\title{
Calibration of the Absolute Efficiency of Well-Type NaI(Tl) Scintillation Detector in 0.121-1.408 MeV Energy Range
}

\author{
Dalal Al Oraini \\ Physics Department, Faculty of Science, Princess Nourah Bint Abdulrahman University, Riyadh 11544-55532, Saudi Arabia \\ Correspondence should be addressed to Dalal Al Oraini; dalalaorainy1335@gmail.com
}

Received 30 September 2017; Accepted 16 November 2017; Published 1 February 2018

Academic Editor: Arkady Serikov

Copyright (C) 2018 Dalal Al Oraini. This is an open access article distributed under the Creative Commons Attribution License, which permits unrestricted use, distribution, and reproduction in any medium, provided the original work is properly cited.

\begin{abstract}
Well-type $\mathrm{NaI}(\mathrm{Tl})$ detectors are beneficial for low-level photon activity measurements because of the near $4 \pi$ solid angle that can be gained with them. The detection efficiency can differ with the source-to-detector system geometries, the absorption of the photon in the detector material, and attenuation layers in front of the detector face. For these purposes, the absolute efficiency and the coincidence corrections of the well-type sodium iodide detector have been measured at $0.121-1.408 \mathrm{MeV}$ energy range (obtained from ${ }^{152} \mathrm{Eu},{ }^{137} \mathrm{Cs}$, and ${ }^{60} \mathrm{Co}$ radioactive isotopes). The covenant between the experimental (present work) and the published theoretical values is good, with the high discrepancies being less than $1 \%$.
\end{abstract}

\section{Theoretical Viewpoint}

In the case where an isotropic radiating axial point source is in the detector well-cavity at a distance, $h$, from cavity bottom (see Figure 1), the path of the photon is well defined by the geometrical solid angle, $\Omega$, subtended by the source-to-detector system at the point of entry. The solid angle is given as

$$
\Omega=\int_{\phi} \int_{\theta} \sin (\theta) d \theta d \phi
$$

The usage of well-type gamma spectrometry systems is useful for low-level gamma activity measurements. To measure the sample's activity, the photopeak efficiency (FEPE) of the detector for each photon energy is needed. This is usually obtained by the efficiency calibration by the use of standard radioactive sources of identical geometrical shape and dimensions with the samples under study [1]. However, the MC simulations consider the detailed characteristics of the source-to-detector system in calculating the photopeak efficiency. This approach (MC) is inadequate in its accuracy because of the inaccuracy in the parameters accompanying the detector's geometrical dimensions and the structure of the sample [2]. The accuracy is also affected by the uncertainty in nuclear data and the calculation uncertainties of the MC code [3], but these are likely to be as important as the parameters linked with the detector's geometrical dimensions and the material composition of the sample. The physical dimensions provided by suppliers are usually unsatisfactory for accurate efficiency calculations because any slight change in some of these geometrical parameters can cause significant deviations from experimental values. Several studies of the response of $\gamma$-ray spectrometers using MC simulations have been published. Most of the authors report agreement with experimentally obtained efficiency values within $10 \%$. One useful way to stun these complications is the use of the straightforward direct mathematical method [4-17] and the experimental measurements.

For the polar $(\theta)$ and azimuthal $(\varphi)$ angles, the azimuthal angle, $\varphi$, earns the values from 0 to $2 \pi$, while the polar angle, $(\theta)$, earns four different values built on the source-to-detector configuration.

$$
\begin{aligned}
& \theta_{1}=\tan ^{-1}\left(\frac{R_{1}}{h}\right), \\
& \theta_{2}=\pi-\tan ^{-1}\left(\frac{R_{1}}{S-h}\right), \\
& \theta_{3}=\pi-\tan ^{-1}\left(\frac{R_{2}}{S-h}\right), \\
& \theta_{4}=\tan ^{-1}\left(\frac{R_{2}}{L-S+h}\right),
\end{aligned}
$$




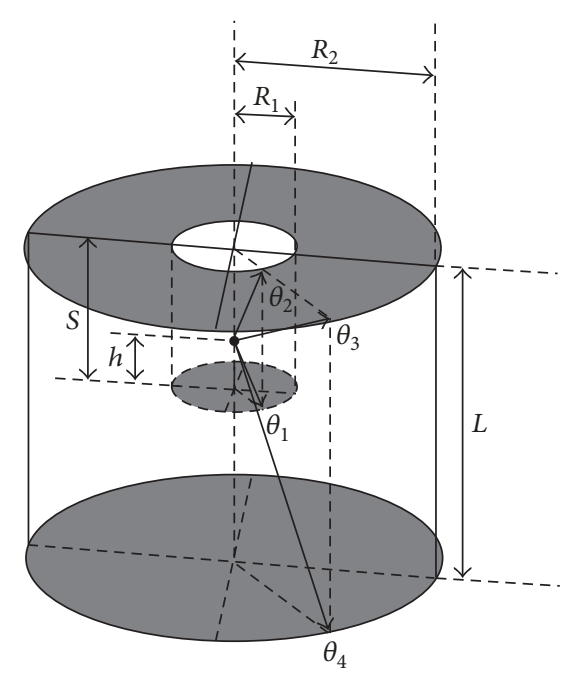

FIGURE 1: Well-type detector with an isotropic radiating axial point source in the detector well-cavity.

where $R_{1}$ is the detector well-cavity inner radius, $R_{2}$ is the outer radius, $S$ is the well depth, and $L$ is the detector side length, as exposed in Figure 1. There are two main cases to determine the detector efficiency depending on the sourceto-detector well bottom, $h$; these two main cases contain five subcases. The photons have different five possible path lengths affording to the photon entrance and emittance point from the detector body. These path lengths are represented in Figure 2 and are specified by equation (3) as follows:

$$
\begin{aligned}
& d_{1}=\frac{L-S}{\cos (\theta)}, \\
& d_{2}=\frac{R_{2}}{\sin (\theta)}-\frac{h}{\cos (\theta)}, \\
& d_{3}=\frac{L-S+h}{\cos (\theta)}-\frac{R_{1}}{\sin (\theta)}, \\
& d_{4}=\frac{R_{2}}{\sin (\theta)}-\frac{R_{1}}{\sin (\theta)}, \\
& d_{5}=\frac{S-h}{\cos (\theta)}-\frac{R_{1}}{\sin (\theta)} .
\end{aligned}
$$

(a) In case of $\theta_{2}>\theta_{3}>\theta_{1}>\theta_{4}$ existing in Figure 2(a), the four possible path lengths have been initiated to be $\left(d_{1}, d_{2}, d_{4}\right.$, and $\left.d_{5}\right)$ and the detection efficiency will be specified by

$$
\begin{aligned}
\varepsilon= & \frac{1}{4 \pi} \int_{0}^{2 \pi}\left(\int_{0}^{\theta_{4}} f_{\text {att }} f_{1} \sin (\theta) d \theta\right. \\
& +\int_{\theta_{4}}^{\theta_{1}} f_{\text {att }} f_{2} \sin (\theta) d \theta+\int_{\theta_{1}}^{\theta_{3}} f_{\text {att }} f_{4} \sin (\theta) d \theta \\
& \left.+\int_{\theta_{3}}^{\theta_{2}} f_{\text {att }} f_{5} \sin (\theta) d \theta\right) d \varphi .
\end{aligned}
$$

(b) In case of $\theta_{2}>\theta_{3}>\theta_{4}>\theta_{1}$ existing in Figure 2(b), the four possible path lengths have been initiated to be $d_{1}, d_{3}, d_{4}$, and $d_{6}$ and the detection efficiency will be specified by

$$
\begin{aligned}
\varepsilon= & \frac{1}{4 \pi} \int_{0}^{2 \pi}\left(\int_{0}^{\theta_{1}} f_{\text {att }} f_{1} \sin (\theta) d \theta\right. \\
& +\int_{\theta_{1}}^{\theta_{4}} f_{\text {att }} f_{3} \sin (\theta) d \theta+\int_{\theta_{4}}^{\theta_{3}} f_{\text {att }} f_{4} \sin (\theta) d \theta \\
& \left.+\int_{\theta_{3}}^{\theta_{2}} f_{\text {att }} f_{5} \sin (\theta) d \theta\right) d \varphi,
\end{aligned}
$$

where

$$
f_{\text {att }}=1-e^{-\mu \cdot d_{i}}, \quad i=1-5,
$$

where $\mu$ is the detector attenuation coefficient for a photon with energy, $E_{\gamma}$, and $d_{i}$ are the possible path lengths covered by the photons in the detector. Meanwhile the factor $f_{\text {att }}$ is the attenuation factor for the absorbing layers with attenuation coefficient $\mu_{1}, \mu_{2}, \ldots, \mu_{n}$ and with the thickness $t_{1}, t_{2}, \ldots, t_{n}$ in front of the detector face and it is described as

$$
f_{\text {att }}=e^{-\sum_{i=1}^{n} \mu_{i} \delta_{i}}
$$

where

$$
\begin{aligned}
& \delta_{i}=\left(\frac{t_{i}}{\cos \theta}\right) \quad \text { for the horizontal absorber layers, } \\
& \delta_{i}=\left(\frac{t_{i}}{\sin \theta}\right) \quad \text { for the vertical absorber layers. }
\end{aligned}
$$

\section{Experimental Setup}

The $7.62 \times 7.62 \mathrm{~cm}^{2}$ well-type sodium iodide detector, model number 802 , made by CANBERRA Company was used (see Figure 3).

The detector was mounted vertically, the cathode to anode voltage was equal to $+600 \mathrm{~V} \mathrm{dc}$, the dynode to dynode was $+80 \mathrm{~V} \mathrm{dc}$, the cathode to dynode was $+150 \mathrm{~V} \mathrm{dc}$, and the total weight was $1.8 \mathrm{~kg}$. The detector dimensions were given as $0.5 \mathrm{~mm} \mathrm{Al}$ end cap thickness, $2.5 \mathrm{~mm} \mathrm{Al} 2 \mathrm{O} 3$ face reflector layer, $1.85 \mathrm{~mm} \mathrm{Al2O} 3$ side reflector layer, $8.33 \mathrm{~mm}$ cavity radius, and 49.87 cavity depth. The detector energy resolution (FWHM) was $9 \%$ at the $661 \mathrm{keV} \gamma$-ray line of $137 \mathrm{Cs}$ source ground on the manufactory certificate and the shaping mode was Gaussian. The detector was coupled to a CANBERRA data acquisition system (Osprey ${ }^{\mathrm{TM}}$ Base) applying a Genie 2000 analysis software, with many functions including peak area determination. The type of the used sources is radioactive point sources ${ }^{152} \mathrm{Eu},{ }^{137} \mathrm{Cs}$, and ${ }^{60} \mathrm{Co}$ (see Tables 1 and 2 ).

The photopeak efficiencies were obtained experimentally by using (9) as follows:

$$
\varepsilon(E)=\frac{N(E)}{t \cdot A_{S} \cdot P(E)} \prod C_{i},
$$

where $N(E)$ is the counts number in the photopeak (obtained using Genie 2000 software), $t$ is the time of measurement 


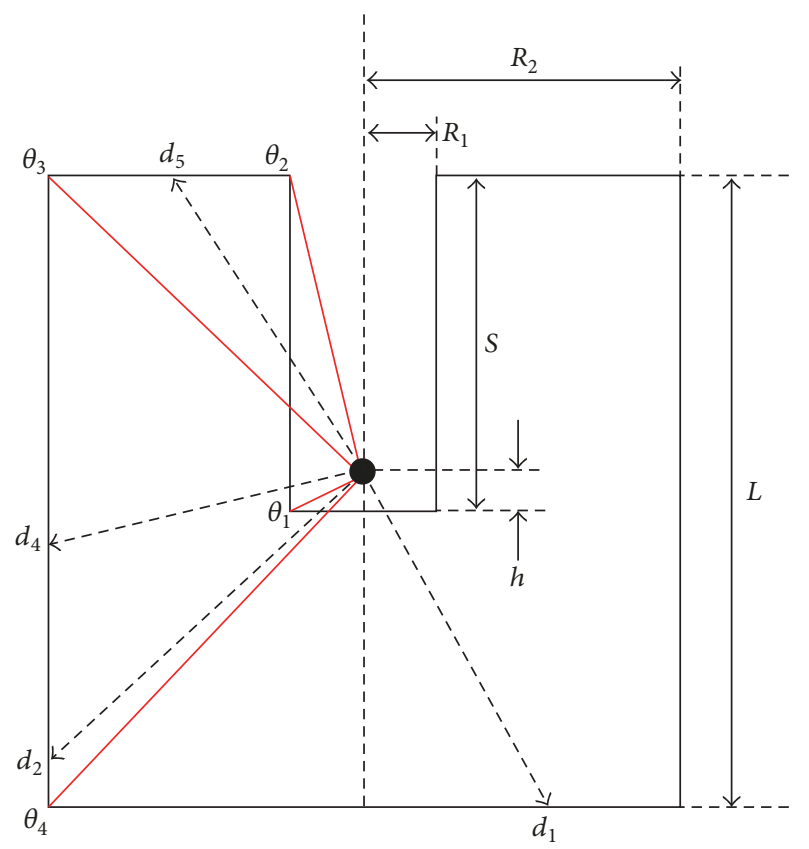

(a) Case 1. $\theta_{2}>\theta_{3}>\theta_{1}>\theta_{4}$

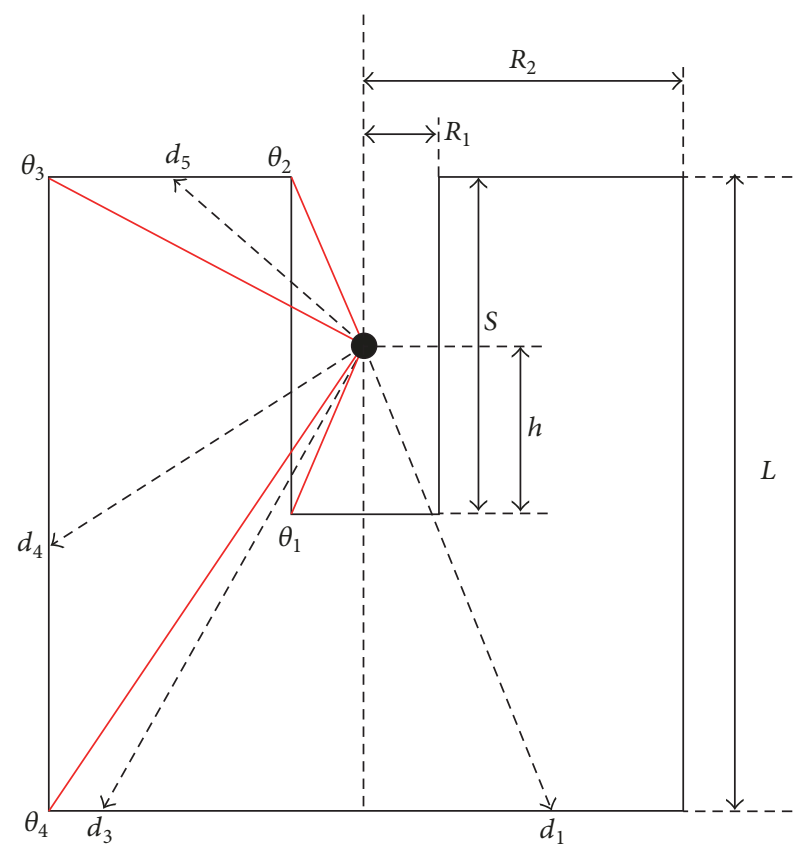

(b) Case 2. $\theta_{2}>\theta_{3}>\theta_{4}>\theta_{1}$

FIgURE 2: The two possible cases of the photon path lengths.

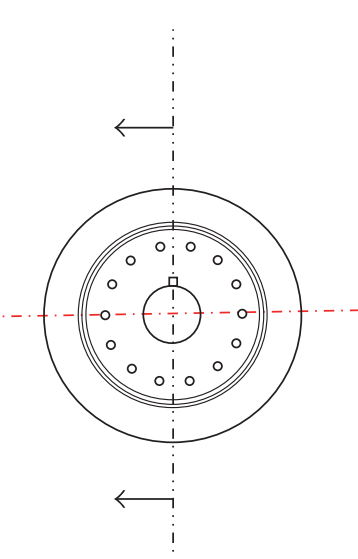

(1) Crystal $7.62 \times 7.62 \mathrm{~cm}^{2} \mathrm{NaI}(\mathrm{Tl})$

(2) 3.0 photomultiplier tube

(3) Bicron proprietary optical coupling

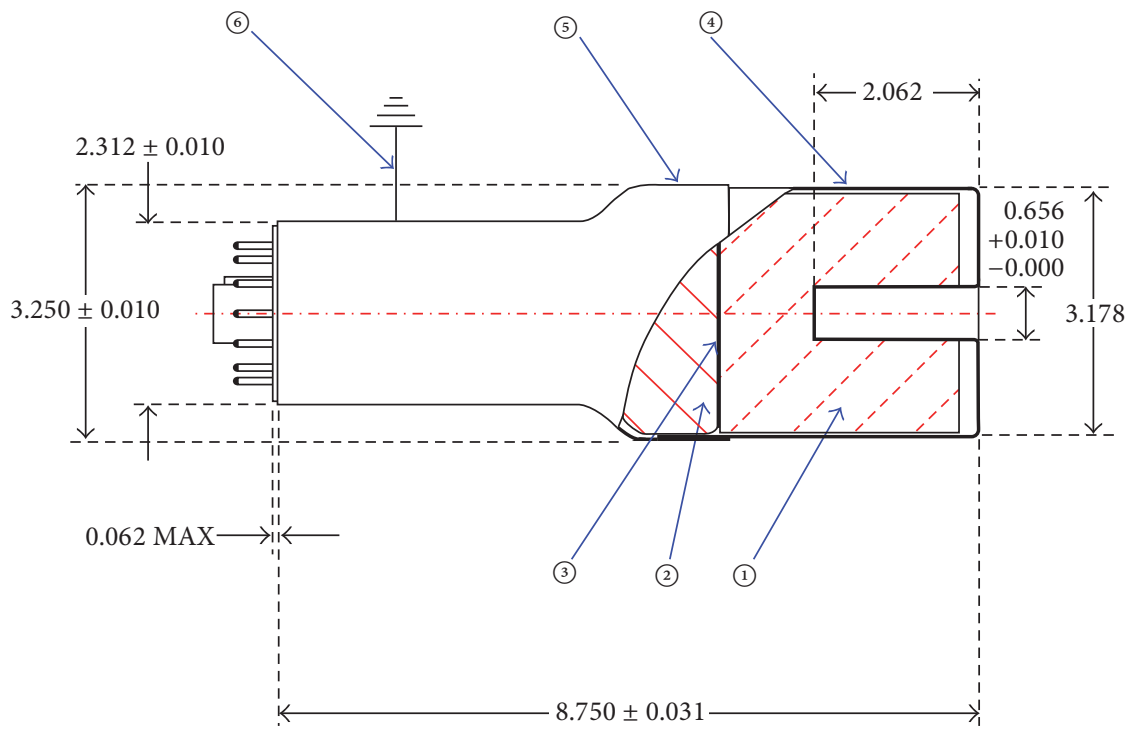

(4) Aluminum housing with well: chrome finish

(5) MU-metal light shield: chrome finish

(6) Light shield grounded to pin 14 (cathode) performance: PHR $\leq 9.0 \%$ for Cs 137

FIGURE 3: The manufactory diagram of $7.62 \times 7.62 \mathrm{~cm}^{2}$ well-type $\mathrm{NaI}(\mathrm{Tl})$ scintillation detector.

TABLE 1: PTB radioactive sources activities and their uncertainties.

\begin{tabular}{lcc}
\hline PTB & Activity & Uncertainty \\
Nuclide & $\mathrm{kBq}$ & $\mathrm{kBq}$ \\
\hline${ }^{152} \mathrm{Eu}$ & 290.0 & \pm 4.0 \\
${ }^{137} \mathrm{Cs}$ & 385.0 & \pm 4.0 \\
${ }^{60} \mathrm{Co}$ & 212.1 & \pm 1.5 \\
\hline
\end{tabular}

(in seconds), $P(E)$ is the photon branching ratio at energy $E$, $A_{S}$ is the nuclide activity, and $C_{i}$ are the correction factors because of coincidence summing corrections, radionuclide decay, and dead time. The decay correction $\left(C_{d}\right)$ was given by

$$
C_{d}=e^{\lambda \cdot \Delta T},
$$


TABLE 2: Specifications of the radionuclides.

\begin{tabular}{lccc}
\hline $\begin{array}{l}\text { PTB } \\
\text { Nuclide }\end{array}$ & $\begin{array}{c}\text { Energy } \\
\text { keV }\end{array}$ & $\begin{array}{c}\text { Emission } \\
\text { probability } \\
\%\end{array}$ & $\begin{array}{c}\text { Half Life } \\
\text { Days }\end{array}$ \\
\hline & 121.78 & 28.4 & \\
& 244.69 & 7.49 & \\
${ }^{152} \mathrm{Eu}$ & 344.28 & 26.6 & 4943.29 \\
& 778.90 & 12.96 & \\
& 964.13 & 14.0 & 11004.98 \\
\hline${ }^{137} \mathrm{Cs}$ & 1408.01 & 20.87 & 1925.31 \\
${ }^{60} \mathrm{Co}$ & 661.66 & 85.21 & \\
& 1173.23 & 99.9 & \\
\hline
\end{tabular}

where $\lambda$ is the decay constant and $\Delta t$ is the time interval between the source decay time and the run time. The main source of uncertainty in the efficiency calculations was the uncertainties of the activities of the standard source solutions. The uncertainty in the photopeak efficiency, $\sigma_{\varepsilon}$, was given by

$$
\begin{aligned}
& \sigma_{\varepsilon} \\
& =\varepsilon \\
& \cdot \sqrt{\left(\frac{\partial \varepsilon}{\partial A}\right)^{2} \cdot \sigma_{A}^{2}+\left(\frac{\partial \varepsilon}{\partial P}\right)^{2} \cdot \sigma_{P}{ }^{2}+\left(\frac{\partial \varepsilon}{\partial N}\right)^{2} \cdot \sigma_{N}{ }^{2}},
\end{aligned}
$$

where the uncertainties $\sigma_{A}, \sigma_{P}$, and, $\sigma_{N}$ are linked with the quantities $A_{S}, P(E)$, and $N(E)$, respectively. The percentage of deviation among the calculated and measured efficiencies is given by

$$
\Delta \%=\frac{\varepsilon_{\mathrm{Th}}-\varepsilon_{\mathrm{Exp}}}{\varepsilon_{\mathrm{Th}}} \times 100,
$$

where $\varepsilon_{\mathrm{Th}}$ and $\varepsilon_{\mathrm{Exp}}$ are the theoretically and experimentally measured efficiencies, respectively.

\section{Energy Calibrations and Resolution}

The detection system must be calibrated before the use in radiation detection to hide channel number to energy scale. The energy, shape, and efficiency calibration of the $\mathrm{NaI}(\mathrm{Tl})$ well-type detector was a procedure occasionally made to establish the linking between the energy of the photon, the channel number, and the detector efficiency. This process was done by using Osprey Universal Digital Multichannel Analyzer Base for scintillation spectrometry, where after the identification of the energy using standard sources, the efficiency values were calculated considering the probability of disintegration for each energy. The typical energy and shape calibration of the amplitudes from standard $\left({ }^{60} \mathrm{Co}\right.$ and ${ }^{137} \mathrm{Cs}$ ) radioactive sources used for calibration at position $25 \mathrm{~cm}$ are shown in Figures 4 and 5. The NaI(Tl) well-type detector energy resolution was found to be $\sim 6.9 \%$ for $662 \mathrm{keV}$ gammas from ${ }^{137} \mathrm{Cs}$. The relation between the energy and the

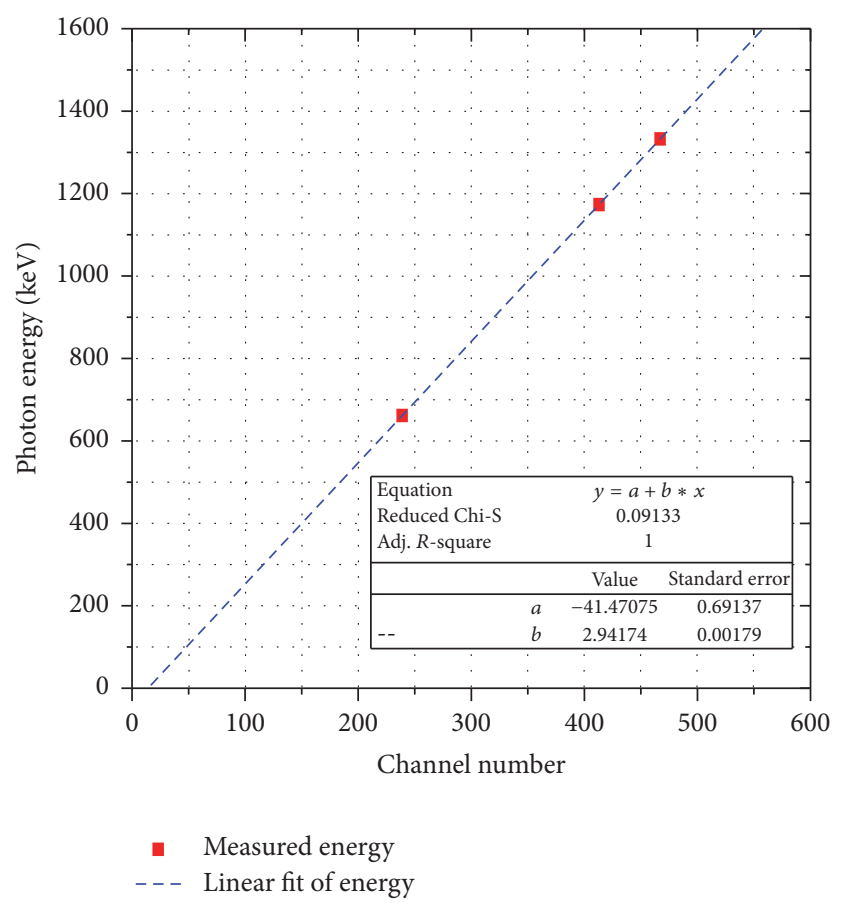

FIGURE 4: The calibration energy curves (measured and fit) using standard point sources $\left({ }^{60} \mathrm{Co}\right.$ and $\left.{ }^{137} \mathrm{Cs}\right)$ with $\mathrm{NaI}(\mathrm{Tl})$ well-type detector.

channel number $(X)$ is a first-degree polynomial and can be given by

$$
E=a+b \cdot X
$$

where $E$ is the $\gamma$-ray energy in $\mathrm{keV}$ and $X$ is the spectral channel number of the center of the peak corresponding to the energy $E$, while the parameters $a=-41.47075$ and $b=2.94174$ are constants to be calculated by the energy calibration process.

The resolution (FWHM) calibration curve was established as a role to pronounce the peak width against the spectral energy. It is considered as significant limit illustrating the system act in separating different photon emissions in an energy range, The relation between the FWHM and the energy is a first-degree polynomial and can be given by

$$
\text { FWHM }[\mathrm{keV}]=a+b \cdot E,
$$

while the parameters $a=8.70616$ and $b=-0.00269$ are constants to be calculated by the shape calibration process.

\section{Results and Conclusions}

The well-type sodium iodide detector photopeak efficiency (FEPE) was measured and compared with the calculated values. The disparity of efficiency with the photon energy was also investigated. The overall efficiency curves are obtained by fitting a polynomial logarithmic function of third order 


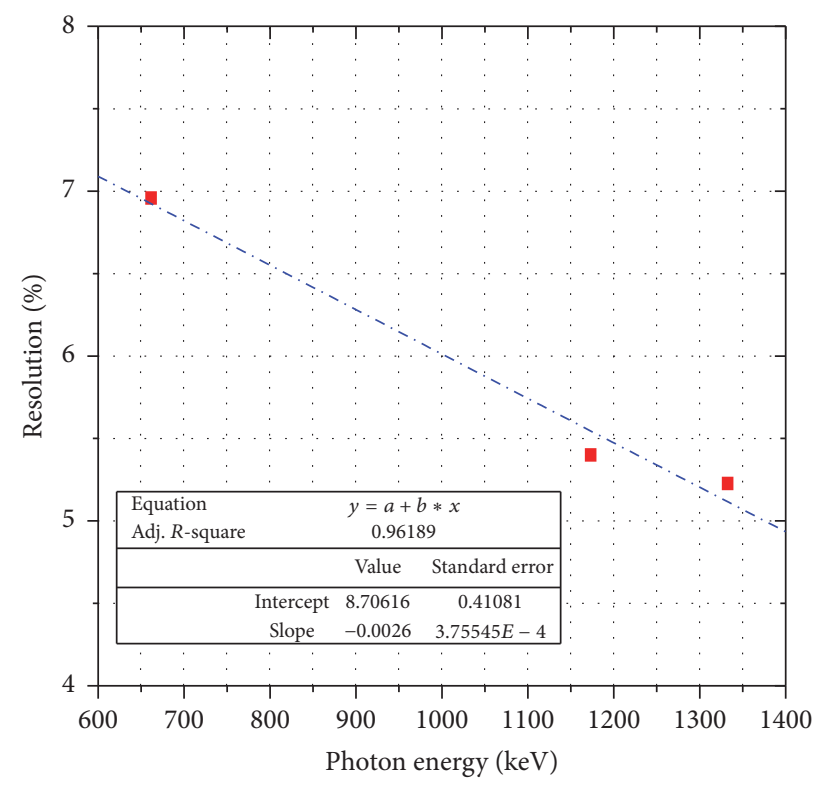

- Measured resolution

-. . Linear fit of resolution

FIGURE 5: The calibration energy curves (measured and fit) using standard point sources $\left({ }^{60} \mathrm{Co}\right.$ and $\left.{ }^{137} \mathrm{Cs}\right)$ with $\mathrm{NaI}(\mathrm{Tl})$ well-type detector.

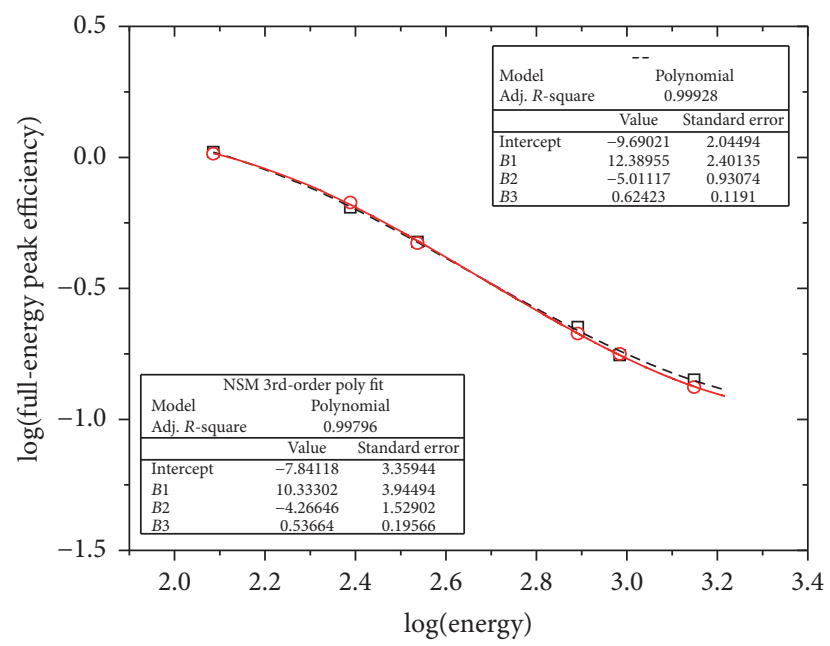

FIgURE 6: The variation of the calculated photopeak efficiency of $7.62 \times 7.62 \mathrm{~cm}^{2} \mathrm{NaI}(\mathrm{Tl})$ well-type sodium iodide detector as a function of photon energy. Square symbols are the experimental present work; red solid line and its circles represent the values calculated using Abbas formulae [4] and dashed line is the fitting.

for the photopeak efficiencies points, using a nonlinear least square fit built on the following equation:

$$
\log (\varepsilon)=\sum_{i=0}^{3}\left(a_{i} \log (E)^{i}\right),
$$

where $a_{i}$ are the coefficients to be determined by the calculations and $\varepsilon$ is the photopeak efficiency (FEPE) of the well-type sodium iodide detectors at energy E. As given in Figure 6, the variation of the experimentally measured and calculated photopeak efficiencies of the well-type scintillation detector as a function of the energy of photon can come into sight. The behavior of these curves was based on using a vile filled with small amount of ${ }^{152}$ Eu aqueous solution of a well-known activity and measured inside the well-type detectors cavity. Results based on ${ }^{152} \mathrm{Eu}$ sources indicate a good covenant between the measured photopeak efficiency values and the theoretical ones [4], with the high discrepancies being less than $1 \%$.

\section{Conflicts of Interest}

The author declares that there are no conflicts of interest regarding the publication of this paper.

\section{References}

[1] M. J. Vargas, N. C. Diaz, and D. P. Sánchez, "Efficiency transfer in the calibration of a coaxial p-type HpGe detector using the Monte Carlo method," Applied Radiation and Isotopes, vol. 58, no. 6, pp. 707-712, 2003.

[2] M. García-Talavera, H. Neder, M. J. Daza, and B. Quintana, "Towards a proper modeling of detector and source characteristics in Monte Carlo simulations," Applied Radiation and Isotopes, vol. 52, no. 3, pp. 777-783, 2000.

[3] J.-M. Laborie, G. Le Petit, D. Abt, and M. Girard, "Monte Carlo calculation of the efficiency calibration curve and coincidencesumming corrections in low-level gamma-ray spectrometry using well-type HPGe detectors," Applied Radiation and Isotopes, vol. 53, no. 1-2, pp. 57-62, 2000.

[4] M. I. Abbas, "Analytical formulae for well-type $\mathrm{NaI}$ (Tl) and HPGe detectors efficiency computation," Applied Radiation and Isotopes, vol. 55, no. 2, pp. 245-252, 2001.

[5] M. I. Abbas, "Direct mathematical method for calculating fullenergy peak efficiency and coincidence corrections of HPGe detectors for extended sources," Nuclear Instruments and Methods in Physics Research Section B, vol. 256, no. 1, pp. 554-557, 2007.

[6] S. S. Nafee and M. I. Abbas, "A theoretical approach to calibrate radiation portal monitor (RPM) systems," Applied Radiation and Isotopes, vol. 66, no. 10, pp. 1474-1477, 2008.

[7] S. S. Nafee and M. I. Abbas, "Calibration of closed-end HPGe detectors using bar (Parallelepiped) sources," Nuclear Instruments and Methods in Physics Research Section A, vol. 592, no. 1-2, pp. 80-87, 2008.

[8] M. I. Abbas, "Analytical approach to calculate the efficiency of $4 \pi \mathrm{NaI}(\mathrm{Tl})$ gamma-ray detectors for extended sources," Nuclear Instruments and Methods in Physics Research Section A, vol. 615, no. 1, pp. 48-52, 2010.

[9] M. I. Abbas, "A new analytical method to calibrate cylindrical phoswich and $\mathrm{LaBr}_{3}(\mathrm{Ce})$ scintillation detectors," Nuclear Instruments and Methods in Physics Research Section A, vol. 621, no. 1-3, pp. 413-418, 2010.

[10] M. I. Abbas, "Analytical formulae for borehole scintillation detectors efficiency calibration," Nuclear Instruments and Methods in Physics Research Section A, vol. 622, no. 1, pp. 171-175, 2010.

[11] M. I. Abbas and S. Noureddeen, "Analytical expression to calculate total and full-energy peak efficiencies for cylindrical 
phoswich and lanthanum bromide scintillation detectors," Radiation Measurements, vol. 46, no. 4, pp. 440-445, 2011.

[12] M. S. Badawi, I. Ruskov, M. M. Gouda et al., "A numerical approach to calculate the full-energy peak efficiency of HPGe well-type detectors using the effective solid angle ratio," Journal of Instrumentation, vol. 9, no. 7, p. P07030, 2014.

[13] M. I. Abbas, M. S. Badawi, I. N. Ruskov et al., "Calibration of a single hexagonal $\mathrm{NaI}(\mathrm{Tl})$ detector using a new numerical method based on the efficiency transfer method," Nuclear Instruments and Methods in Physics Research Section A, vol. 771, pp. 110-114, 2015.

[14] M. I. Abbas, S. Hammoud, T. Ibrahim, and M. Sakr, "Analytical formulae to calculate the solid angle subtended at an arbitrarily positioned point source by an elliptical radiation detector," Nuclear Instruments and Methods in Physics Research Section A, vol. 771, pp. 121-125, 2015.

[15] A. Hamzawy, D. N. Grozdanov, M. S. Badawi et al., "New numerical simulation method to calibrate the regular hexagonal $\mathrm{NaI}(\mathrm{Tl})$ detector with radioactive point sources situated nonaxial," Review of Scientific Instruments, vol. 87, no. 11, Article ID 115105, 2016.

[16] M. I. Abbas, M. M. Gouda, M. S. Badawi, and A. M. El-Khatib, "Direct mathematical solutions for the gamma-ray detectors geometrical andtotal efficiencies integrable formulae," Journal of Engineering Science \& Technology, vol. 12, no. 3, pp. 701-715, 2017.

[17] S. F. Noureldine, M. S. Badawi, and M. I. Abbas, "A hybrid analytical-numerical method for efficiency calculations of spherical scintillation $\mathrm{NaI}(\mathrm{Tl})$ detectors and arbitrarily located point sources," Nuclear Technology and Radiation Protection, vol. 32, no. 2, pp. 140-147, 2017. 

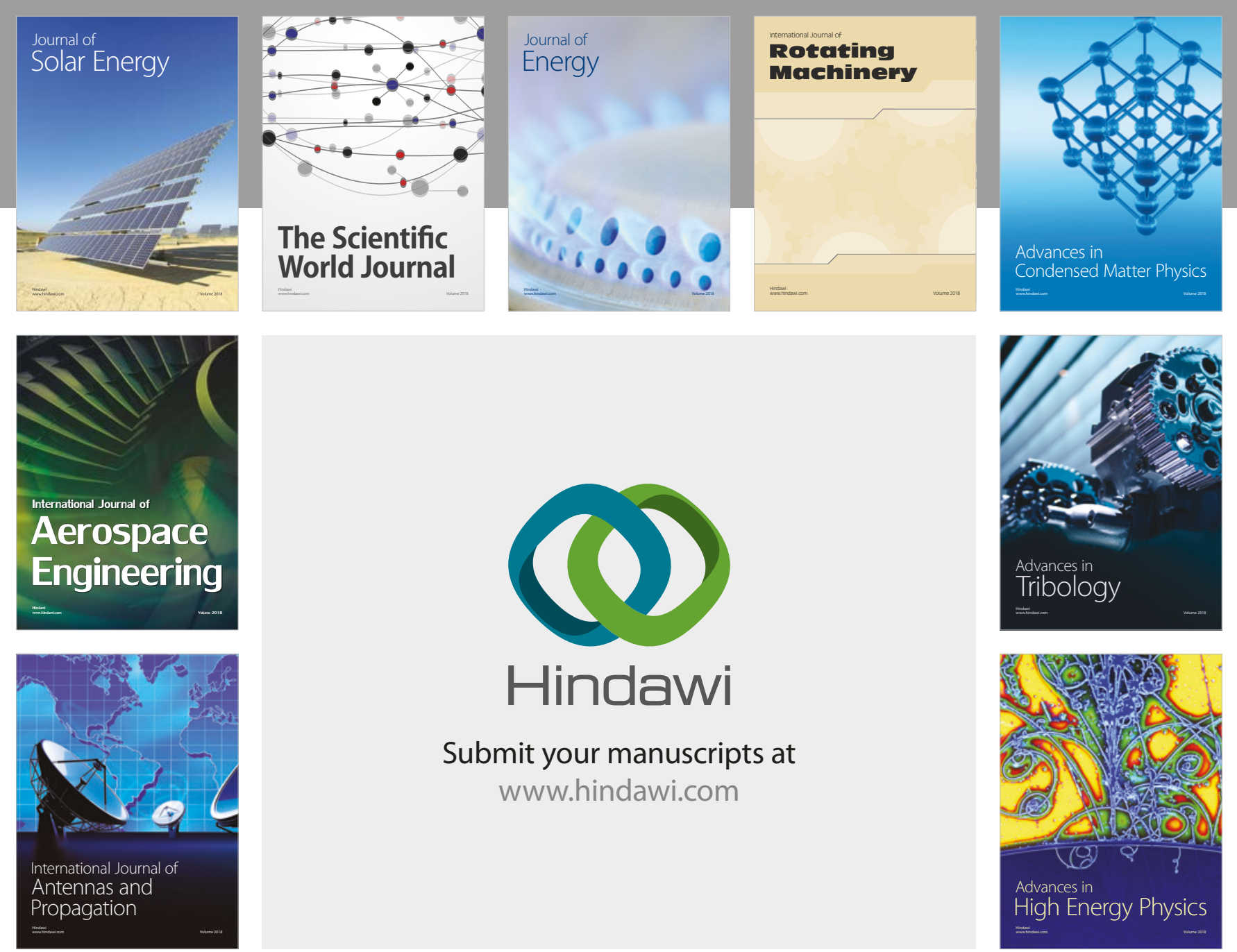

Submit your manuscripts at

www.hindawi.com
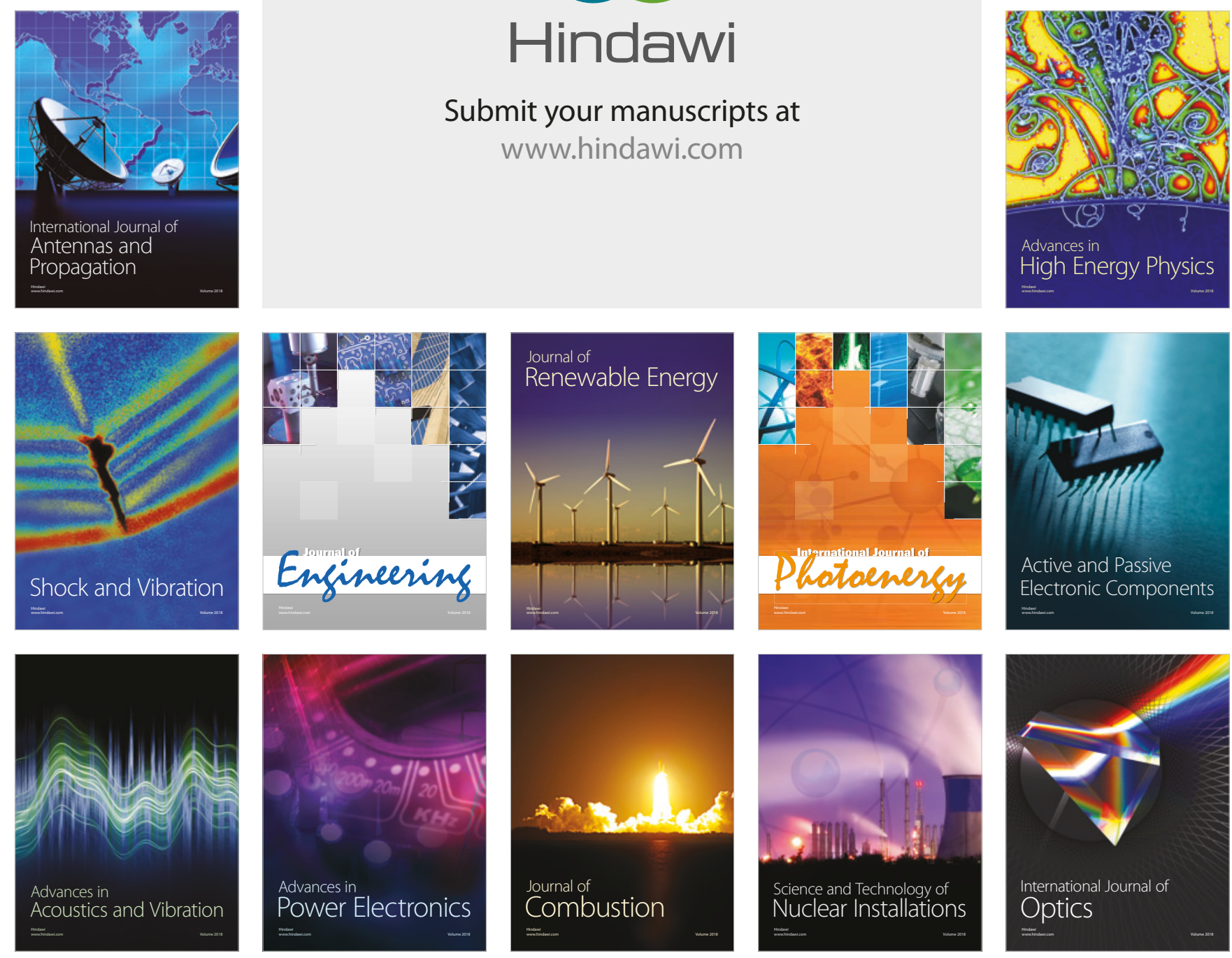